The University of Maine

DigitalCommons@UMaine

Spatial Informatics Faculty Scholarship

Spatial Informatics

2014

\title{
Evaluation of Non-Visual Panning operations using Touch-Screen Devices
}

\author{
Nicholas A. Giudice \\ University of Maine - Main, nicholas.giudice@maine.edu \\ Hari Prasath Palani \\ University of Maine - Main
}

Follow this and additional works at: https://digitalcommons.library.umaine.edu/sie_facpub

Part of the Graphics and Human Computer Interfaces Commons

\section{Repository Citation}

Giudice, Nicholas A. and Palani, Hari Prasath, "Evaluation of Non-Visual Panning operations using Touch-Screen Devices" (2014). Spatial Informatics Faculty Scholarship. 3.

https://digitalcommons.library.umaine.edu/sie_facpub/3

This Conference Proceeding is brought to you for free and open access by DigitalCommons@UMaine. It has been accepted for inclusion in Spatial Informatics Faculty Scholarship by an authorized administrator of DigitalCommons@UMaine. For more information, please contact um.library.technical.services@maine.edu. 


\section{Evaluation of Non-Visual Panning operations using Touch-Screen Devices}

\author{
HariPrasath Palani \\ School of Computing and Information Science \\ University of Maine, \\ 5711 Boardman Hall, Orono, Maine, USA 04469 \\ hariprasath.palani@maine.edu
}

\author{
Nicholas A. Giudice \\ School of Computing and Information Science \\ University of Maine, \\ 5711 Boardman Hall, Orono, Maine, USA 04469 \\ nicholas.giudice@maine.edu
}

\begin{abstract}
This paper summarizes the implementation, evaluation, and usability of non-visual panning operations for accessing graphics rendered on touch screen devices. Four novel non-visual panning techniques were implemented and experimentally evaluated on our experimental prototype, called a Vibro-Audio Interface (VAI), which provides completely non-visual access to graphical information using vibration, audio, and kinesthetic cues on a commercial touch screen device. This demonstration will provide an overview of our system's functionalities and will discuss the necessity for developing non-visual panning operations enabling visually-impaired people access to large-format graphics (such as maps and floor plans).
\end{abstract}

\section{Categories and Subject Descriptors}

H.5.2 [User Interfaces]: Haptic I/O, Auditory (non-speech) feedback, Evaluation/methodology; H.5.4 [Hypertext/ Hypermedia]: Navigation; K.4.2 [Social Issues]: Assistive technologies for persons with disabilities

\section{Keywords}

Accessibility (blind and visually-impaired); Assistive Technology; Touch-screens; Haptic cues; Auditory Cues; Vibro-Audio Interface; non-visual maps.

\section{INTRODUCTION}

An increasing amount of information content used in the workplace, educational settings, and for everyday living is presented in graphical form. However, the visual nature of this graphical material prevents numerous visually-impaired users from accessing this key information. Although several approaches have been advanced for providing non-visual access to graphical material, they are often very expensive and have not experienced broad market penetration or end-user acceptance. As this demographic is estimated to number around 285 million people worldwide [11], the need for developing devices that are both affordable and usable for accessing non-visual graphics is critical for educational, social, and vocational purposes. With the advent and proliferation of touch-screen devices (such as smartphones), several R\&D projects have focused on developing touch based

\footnotetext{
Permission to make digital or hard copies of all or part of this work for personal or classroom use is granted without fee provided that copies are not made or distributed for profit or commercial advantage and that copies bear this notice and the full citation on the first page. Copyrights for third-party components of this work must be honored. For all other uses, contact the Owner/Author.

Copyright is held by the owner/author(s)

ASSETS'14, October 20-22, 2014, Rochester, New York, USA.

ACM 978-1-4503-2720-6/14/10.

http://dx.doi.org/10.1145/2661334.2661336
}

accessibility solutions. Most of these solutions involve incorporation of vibro-tactile and/or auditory feedback to convey patterns on the touch-screen. However, these solutions are generally used for research purposes, meaning that they are not commercially available. Also, many of these R\&D efforts emphasize technical design features and algorithms as opposed to empirical experiments and behavioral evaluations $[1,6,8]$. In addition, touch has a very coarse spatial resolution when compared to vision $[2,4]$, which causes restrictions in translating visual representations to tactile representations. These shortcomings have led to a huge information gap in accessing graphical information for visually-impaired persons. To bridge this gap, the tactile representation should be as functionally equivalent as possible with the visual representation by maintaining the spatial and geometric properties of the original rendering. We addressed these issues in our earlier work $[1,6]$ by developing and evaluating a novel touch-screen based interface called a Vibro-Audio Interface (VAI). VAI was developed based on empirical results and usability guidelines derived from human behavioral evaluations [8]. Findings from these experiments provided strong evidence that the VAI is a viable solution for accurate learning of graphical materials on touch-screen devices.

\section{NON-VISUAL PANNING}

One limitation of commercial touch-screen devices is their limited display size. This necessitates the use of panning operations to access large-format graphics. Use of panning is common for visually-rendered material on portable devices, or even on standard computer monitors. However, this action is not generally done with tactile graphics, which are usually rendered statically on hardcopy tactile output or fixed-format digital displays. Even with approaches that have used dynamic haptic displays and touch screens, non-visual panning has not been widely studied [7, 9, 10]. In order to access large-format graphics, it is necessary to incorporate panning operations for both visual and non-visual interfaces. The need for panning is particularly critical in tactile interfaces owing to the lower spatial resolution of touch and the limited information density of dynamic-tactile displays. For instance, consider a 7 inch touch-screen device ( 170 ppi resolution). A visual interface could render $\sim 776$ vertical lines of width at $0.116 \mathrm{~mm}$ (width that can be perceived by the naked eye at a viewing distance of $\sim 400 \mathrm{~mm}$ ) on this display real estate. By contrast, the optimal line width for perceiving vibro-tactile lines on a touchscreen is $0.35 \mathrm{inch}(8.89 \mathrm{~mm})[1,8]$. This means that for the same screen size, only 10 tactile lines could be rendered, a reduction of information of over 7 orders of magnitude. Because of this huge difference in spatial resolution, it frequently requires multiple displays of information using a non-visual interface to convey the same content of a single visual display. Thus, effectively incorporating panning operations are crucial for any touch-screen based interfaces using vibro-tactile cuing. 


\section{EXPERIMENTAL EVALUATION}

With visual interfaces (such as Google maps), panning is a default feature and sighted users can perform panning operations in many ways, such as using drag, swipe, and other gestures. However, these techniques are almost always visually based and lead to some significant challenges when implemented in a non-visual interface. In order to conceptualize the issue, the reader is invited to try to pan a map using their map interface of choice (e.g., Google map) with their eyes closed. Once panned, the user will likely lose knowledge of their location on the map, as there is no reference between the graphical elements perceived before and after panning. By explanation, tactile perception is serially processed and has a small field of view from a given tactile sample (e.g., exploring the screen with one fingertip). By contrast, vision is highly parallel and has a large field of view (e.g., seeing the whole screen at once). As such, haptic spatial perception can be a challenging process $[3,5,9]$, and unless carefully considered, incorporating additional operations such as panning will likely increase the complexity of accessing graphical materials. Based on empirical studies with the VAI [6], we identified a variety of problems that arise during haptic map learning. For instance, the smooth touch screen surface makes it difficult to stay oriented on the display while tracing graphics [6] and finger transpositions lead to misunderstanding of relationships between the graphical elements [3]. Also, finger location acts as the primary and only orienting reference on the map, which mandates the user to always remember where they are within the given graphic. Accurate orientation is a key design requirement for any tactile based nonvisual interface, as it allows users to develop a reference and integrate graphical elements across time and space. This makes visual panning techniques such as swipe and drag impractical for use in tactile based non-visual interfaces as these actions cause the user to lose their reference finger location after panning. To address these problems, we developed four novel non-visual panning methods and implemented and tested them on the VAI. The panning methods involve use of either single or multi-touch actions, physical buttons (See Figure 1).

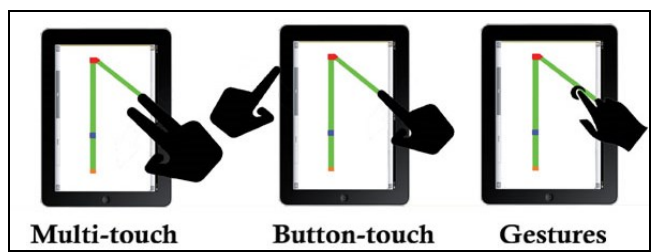

Figure 1: Sample stimuli and different panning methods on a Touch screen.

Experiments were performed on 15 sighted blindfolded participants ( 8 males and 7 females) between the ages of 19 and 29. Inclusion of blindfolded-sighted people is reasonable in this scenario as our focus is on testing the ability to learn and represent non-visual graphics, which are equally perceptible to both blindfolded-sighted and blind groups [6]. A total of five conditions were tested, namely, Two-finger pan, Button-touch pan, Button-Swipe pan, Grip-Tap pan and one without panning. In each condition, participants learned a corridor layout map and performed subsequent spatial testing and reconstruction tasks. The study followed a within-subject design, where a total of 10 performance measures (such as learning time, reconstruction accuracy, etc.) were measured. The performance data for each of the measures were then analyzed using repeated measures ANOVAs and paired-sample t-Tests between the conditions.
Results (see [6]) showed that incorporating panning operations that are optimized for non-visual use do not lead to any detrimental effects on the cognitive representation of the graphical material tested. Indeed, for many of the directional and reconstruction tasks, the performance accuracies with panning were found to actually be better than the control (no-pan) condition, indicating that panning may actually strengthen the learning process. Based on participants' post-test feedback, we found that there was a preference for use of the multi-touch technique to perform non-visual panning. These findings are substantial given the necessity of panning operations for access to large-format graphics on touch-based devices, especially given the lack of other solutions addressing this critical information access problem for visually-impaired people.

\section{ACKNOWLEDGMENTS}

We acknowledge support from NIDRR grant H133S100049 and NSF grant CHS-1425337 on this project.

\section{REFERENCES}

[1] Giudice, N.A., Palani H.P. et al. 2012. Learning non-visual graphical information using a touch-based vibro-audio interface. Proceedings of the 14th international ACM SIGACCESS conference on Computers and accessibility (New York, NY, USA, 2012), 103-110.

[2] Jones, L.A. and Lederman, S.J. 2006. Human hand function. Oxford University Press.

[3] Klatzky, R.L., Giudice, N.A. et al.(in press). Touch-screen technology for the dynamic display of $2 D$ spatial information without vision: Promise and progress. Multisensory Research.

[4] Loomis, J.M. et al. 2012. Sensory substitution of vision: Importance of perceptual and cognitive processing. Assistive Technology for Blindness and Low Vision. R. Manduchi and S. Kurniawan, eds. CRC. 162-191.

[5] Loomis, J.M. et al. 1991. Similarity of Tactual and Visual Picture Recognition with Limited Field of View. Perception. 20 (1991), 167-177.

[6] Palani, H.P. 2013. Making Graphical Information Accessible without Vision using Touch-Based devices. Masters Thesis. University of Maine.

[7] Poppinga, B. et al. 2011. TouchOver Map : Audio-Tactile Exploration of Interactive Maps. In Proceedings of the 12th international conference on Human computer interaction with mobile devices ACM, Stockholm, Sweden. (2011), 545550.

[8] Raja, M.K. 2011. The development and validation of a new smartphone based non-visual spatial interface for learning indoor layouts. Masters Thesis University of Maine.

[9] Rastogi, R. et al. 2013. Intuitive tactile zooming for graphics accessed by individuals who are blind and visually impaired. IEEE transactions on neural systems and rehabilitation engineering : a publication of the IEEE Engineering in Medicine and Biology Society. 21, 4 (Jul. 2013), 655-63.

[10] Su, J. et al. 2010. Timbremap: enabling the visuallyimpaired to use maps on touch-enabled devices. Proceedings of the 12th international conference on Human computer interaction with mobile devices and services (2010), 17-26.

[11] WORLD HEALTH ORGANIZATION 2011. Visual impairment and blindness Fact Sheet. http://www.who.int/mediacentre/factsheets/fs282/en/. 